patients had an ECG in two weeks. In general, documentation of reason for not completing an examination was completed.

Conclusion. We found good compliance with recommendations for physical health assessment. Areas for improvement include better assessment of neurology and more thorough blood tests.

Recommended physical health examination for new admissions is not outlined in SABP policy. We recommend the following:

GCS/level of consciousness, cardiovascular, respiratory, abdominal, and neurological examinations, and baseline observations.

ECG should be a requirement of admission. In order to facilitate this, staff need to be trained to perform ECGs.

NICE guidelines refer to HBAlc rather than glucose, which should be reflected in SABP policy.

\section{Basic clinical equipment for physical health assessment in mid Essex inpatient units}

Hesham Abdelkhalek*, Tamsin Griffiths, Kim Motley, Raisa Ramjan and Sophia Harrop

Essex Partnership University NHS Foundation Trust ${ }^{\star}$ Corresponding author.

doi: 10.1192/bjo.2021.806

Aims. It is trust policy that the Basic Clinical Equipment for Physical Health Assessment should be available on each unit. The standard for this audit is therefore $100 \%$ completion.

Background. This was a cross-sectional study of six mental health units across Mid Essex. We audited equipment and consumables in comparison to trust policies. For the purpose of the audit we designed an audit tool.

Method. Overall compliance across all wards for all audited items was $77.5 \%(64.9 \%-87.5 \%)$. Average compliance for equipment provision $83.3 \%(73.9 \%-91.3 \%)$ was greater than that for consumables $72.1 \%(58.8 \%-82.4 \%)$.

Result. When looking at the compliance on each unit separately, our data show that no unit has met the standard of $100 \%$ for equipment or consumables. From all units, one of the two older adults' inpatient units had the highest overall compliance and highest compliance for consumables at $87.5 \%$ and $82.4 \%$ respectively while the perinatal unit had the lowest overall compliance and lowest compliance for consumables at $64.9 \%$ and 58.8 respectively. For the equipment compliance, intensive care unit and one of the older adults tied for the highest compliance at 91.3\% while male inpatient unit and perinatal inpatient unit were tied the lowest compliance at $73.9 \%$.

Conclusion. This is an audit to assess the availability of Basic Clinical Equipment for Physical Health Assessment on inpatient units in Mid Essex. With an audit standard of $100 \%$ completion, it shows that overall compliance on all units was $77.5 \%$ which is not meeting our standard.

\section{Audit cycle - VTE risk assessment in inpatient wards in mid Essex}

Hesham Abdelkhalek*, Adel Elagawany, Fiona McDowall, Matthew Leahy, Emily Baker and Nkechi Penberton

Essex Partnership University NHS Foundation Trust ${ }^{\star}$ Corresponding author.

doi: 10.1192/bjo.2021.807

Aims. It is trust policy that the VTE risk assessment should be completed for every patient admitted to wards. The standard for this audit is therefore $100 \%$ completion. We completed the audit in October 2018 and closed the loop in September 2019. Method. This was a cross-sectional study of all patients on all the wards according to patients' list on the electronic system (Paris) on certain date. In the first audit we used an audit tool from a similar audit performed in another area in the trust. For the purpose of re- audit we designed an audit tool to reflect the changes made in the electronic form.

Result. In the re-audit, there was noticeable improvement in the completion rate compared to initial audit (95\% vs. $82 \%$ ); however, there was still under-performance. An interesting observation of the re-audit is that $74 \%$ percent of admissions had VTE risk assessments forms completed on same day of admission or next day compared to only $45 \%$ in previous audit.

Conclusion. When looking at the completion of individual components on the VTE forms there are still some room for improvement as well. For example, in $26 \%$ of the patients there was no documentation about the use of prophylactic anticoagulants before admission compared to $34 \%$ in our previous audit. Also in $7 \%$ of the patients there was no documentation about the outcome of the assessment compared to only $3 \%$ in previous audit.

This is an audit to assess the completion of electronic VTE forms as per trust policy. Following the initial audit we made recommendations to improve completion rate. In the re-audit there was an improvement in total completion rate but we have not met the goal of $100 \%$ yet.

\section{'Comfortable, safe and valued': an analysis of the impact of COVID-19 on Hertfordshire's Community Perinatal Team}

\section{Stephanie Adeyemi* and Sarah Cohen}

Hertfordshire Partnership University NHS Foundation Trust ${ }^{\star}$ Corresponding author.

\section{doi: $10.1192 /$ bjo.2021.808}

Aims. This study aimed to assess the impact that the COVID-19 pandemic has had on the Hertfordshire Community Perinatal Team (CPT) group interventions and the innovations made.

Background. The CPT is a multidisciplinary mental health service that runs three groups: Circle of Security $(\mathrm{CoS})$, Emotional Coping Skills (ECS) and a peer support group - Wellbeing and Lifestyle. The service has received an increase in referrals during the COVID-19 pandemic.

Method. Methods: Team member and client semi structured interviews were conducted with answers transcribed in real time and analysed. Patient clinical records were accessed via PARIS and analysed in order to identify patient demographics within each group and whether these had changed during the pandemic. Clinical outcome measures and client feedback were evaluated to see whether the change in groups is impacting their clinical effectiveness.

Result. Results: Innovations made by the CPT include: groups becoming virtual, launching of the new Circle of Security Group which helps women tackle the 'Ghosts in the Nursery' and strengthen maternal bonds, restructuring existing groups, breakout room forums and incorporating communication platform apps such as Whatsapp. The Wellbeing and Lifestyle Group increased in size and reach (7 women from 7 areas in 2019 vs 12 women from 12 areas in 2021) with an increased retention rate (71\% in 2019 vs $100 \%$ in 2021) and a decreased attrition rate $(29 \%$ in 2019 to $0 \%$ in 2021). The Emotional Coping Skills group experienced similar changes (10 areas represented in 2019 vs 15 different areas in 2021) with an increased 
retention rate (58\% in 2019 vs $100 \%$ in 2021 ) and decreased attrition rate ( $42 \%$ in 2019 vs $0 \%$ in 2021 ).

Conclusion. The Hertfordshire Community Perinatal Team has responded to the pandemic by innovating existing groups and creating new forums; many of which will continue on even after the pandemic ceases. The groups have acted as a lifeline for women breaking up the monotony and isolation of lockdown life and providing an invaluable space for women to be heard.

\section{Waiting list eradication in serious mental illness (SMI) "secondary care" psychology: addressing an NHS blind spot}

Nicola Airey ${ }^{1 \star}$, Zaffer Iqbal $^{2}$ and Sophie Brown ${ }^{3}$

${ }^{1}$ University of Manchester, Greater Manchester Mental Health NHS Foundation Trust; ${ }^{2}$ Navigo Heath and Social Care CiC, University of Hull and ${ }^{3}$ Navigo Health and Social Care CiC, University of Hull ${ }^{*}$ Corresponding author.

doi: 10.1192/bjo.2021.809

Aims. The poster focuses on the reduction, and eventual eradication, of waiting times within a community-based NHS psychology service in the North East of England. The poster aims to demonstrate the effectiveness of strategies implemented within a secondary care psychology service whilst examining patterns of help-seeking behaviour and treatment compliance in those waiting for therapy, and also the care needs of this cohort following a wait for services.

Background. Secondary care waiting lists for psychological therapy, as highlighted by a recent British Medical Association audit, remain a so-called 'blind-spot' in mental health care provision and a national problem. Tackling waiting lists within this sector has been stated as a priority within the Five Year Forward View, however "core ingredients" of waiting list eradication methodologies and the components leading to such, have yet to be disseminated.

Method. A historical audit and follow-up of clinical data were utilised to gather and analyse data of 208 individuals who were seen by the psychology service between October 2014 and March 2016. Result. No significant differences were found between individuals who successfully completed therapy compared to those who disengaged in regard to demographic or epidemiological variables, or mental health service input. Despite lengthy waiting times of up to 3.69 years, waiting time did not significantly impact whether someone engaged with psychological services. Any form of input from psychological services led to a significant reduction in distress, as measured by the CORE-OM. No individuals who completed therapy were re-referred for psychological input at 12-month follow-up. Conclusion. If imposed appropriately over a suitable time-frame evidence-based, effective and efficient needs-led psychological input can be provided whilst eradicating a waiting list and still remaining flexible, formulation-based and person-centred.

\section{Polypharmacy and potentially inappropriate} medications (PIMs) in older adults referred to a memory clinic

Anietie Akpan*, Bruno De Blaquiere, Issadevi Nellaya, Cornelia Termure and Sujoy Mukherjee

West London NHS Trust

${ }^{\star}$ Corresponding author.

doi: 10.1192/bjo.2021.810

Aims. The older adult is more likely to be prescribed a lot of medications (polypharmacy) on account of multi-morbidity and consequently being under the care of several specialists. Adverse drug events and reactions account for significant morbidity and mortality in this population group. Common sequelae include confusional episodes, dementia syndromes, falls, and higher rates of acute hospital admissions.Medications are not routinely reviewed in elderly care. We sought to estimate the prevalence of polypharmacy, and potentially inappropriate medications (e.g. anticholinergics or medications with central anticholinergic effects) in those referred to the Cognitive Impairment and Dementia Service (Elm Lodge), Older Persons Mental Health, West London NHS Trust.

Method. All referrals between 01/10/2020 and 30/11/2020 were screened for medications prescribed. Polypharmacy was defined as prescription of 5 or more medications. Medications with anticholinergic properties were considered examples of Potentially Inappropriate Medications (PIMs). The Anticholinergic Effect on Cognition (AEC) Tool, 'Medichec', was used to identify and rate anticholinergic burden. Anticholinergic load was also compared using the Anticholinergic Burden Scale (ABS).

Result. Total number of patients referred - 193

11 patients excluded due to unavailable/incomplete medication records.

Study number: 182

Polypharmacy:

$79.67 \%(\mathrm{n}=145)$ were prescribed 5 or more medications.

$44.51 \%(\mathrm{n}=81)$ prescribed 5-9 medications.

$23.08 \%(n=42)$ prescribed $10-14$ medications.

$8.79 \%(\mathrm{n}=16)$ prescribed $15-19$ medications.

$1.67 \%(\mathrm{n}=3)$ prescribed more than 20 medications.

Anticholinergics prescribed (AEC Tool):

$37.36 \%(\mathrm{n}=68)$ prescribed an anticholinergic.

$6.59 \%(n=12)$ prescribed more than 1 anticholinergic.

Anticholinergics (ABS):

$29.67 \%(\mathrm{n}=54)$ prescribed an anticholinergic.

$7.699 \%(\mathrm{n}=14)$ prescribed more than 1 anticholinergic.

Conclusion. Polypharmacy and potentially inappropriate prescribing (e.g. anticholinergics) remain widespread within the older adult population. Anticholinergic load was broadly similar with the Anticholinergic Effect on Cognition tool and the Anticholinergic Burden Scale. Increased anticholinergic burden further compounds risks of cognitive impairment, delirium and death.Other categories of Potentially Inappropriate Medications, including those no longer needed, ought to be identified and reviewed. Over-the-counter medications also need to be screened for.

Elimination or reduction of anticholinergic burden may improve quality of life for patients, as well as cost burden on services.

Pharmacovigilance, collaborative working, and regular training are needed across services providing care for the older adult.

Polypharmacy and potentially inappropriate medications (PIMS) in older adults referred to a liaison psychiatry service

Anietie Akpan* and Omolade Longe

West London NHS Trust

${ }^{*}$ Corresponding author.

doi: 10.1192/bjo.2021.811 